\title{
A CHAOTIC FUNCTION WHOSE NONWANDERING SET IS THE CANTOR TERNARY SET
}

\author{
BAU - SEN DU
}

\begin{abstract}
We introduce a continuous function from $[0,1]$ onto itself whose nonwandering set is the Cantor ternary set $C$. This function is also chaotic in the sense of $\mathrm{Li}$ and Yorke, but with the property that almost all points of $[0,1]$ are eventually fixed. Those points of $C$ which are periodic, eventually periodic, asymptotically periodic or recurrent are also explicitly identified.
\end{abstract}

Let $f:[0,1] \rightarrow[0,1]$ be defined by $f(x)=-3 x+1$ if $0 \leqslant x<1 / 3, f(x)=0$ if $1 / 3 \leqslant x \leqslant 2 / 3$, and $f(x)=3 x-2$ if $2 / 3<x \leqslant 1$. Then $x=1 / 13$ is a periodic point of $f(x)$ with minimal period 3 . Continuous functions from $[0,1]$ into itself with a periodic point whose period is not an integral power of 2 have been called chaotic by several people $[\mathbf{1}, 3-5]$. So this function $f(x)$ is chaotic in that sense. In the sequel, $f(x)$ is always the above-quoted function.

Recall that the Cantor ternary set $C$ is obtained by first removing the (open) middle third $(1 / 3,2 / 3)$ from $[0,1]$, then removing the (open) middle thirds $(1 / 9,2 / 9)$ and $(7 / 9,8 / 9)$ of the remaining 2 intervals, and so on. For our function $f(x)$, it is easy to see that $f(x)$ maps the (closed) middle third [1/3,2/3] of $[0,1]$ onto the (unstable) fixed point $x=1$ and $f^{2}(x)$ maps the (closed) middle thirds $[1 / 9,2 / 9]$ and $[7 / 9,8 / 9]$ of the remaining 2 intervals (in addition to the first closed middle third [1/3,2/3]) onto the fixed point $x=1$, and so on. Since the Cantor set $C$ has (Lebesgue) measure zero, almost all points of $[0,1]$ are mapped onto the same and unstable fixed point $x=1$ by $f(x)$ after only finitely many iterations. Therefore, from a physical point of view, this function $f(x)$ is not chaotic after all (see [2] for more of this). This seems to suggest that chaotic functions should be further classified (see [4] also).

From the above argument, we also obtain that the Cantor ternary set $C$ is invariant under $f(x)$ and the nonwandering set $\Omega$ of $f(x)$ is a subset of $C$. In this note, we identify those points of $\Omega$ which are periodic, eventually periodic, asymptotically periodic or recurrent. As a consequence, we obtain that $\bar{P}=\bar{R}=\Omega=C$, where $P$ ( $R$, respectively) is the set of all periodic (recurrent, respectively) points of $f(x)$.

For every real number $x$ in the Cantor ternary set $C$, there is a unique ternary expansion. That is, $x=\sum_{n=1}^{\infty}\left(a_{n}\right) /\left(3^{n}\right)$, where $a_{n}=0$ or 2 for all $n \geqslant 1$. We shall write $x=a_{1} a_{2} a_{3} \cdots$ from now on. In the sequel, we only consider those points of

Received by the editors March 26, 1983 and, in revised form, October 31, 1983.

1980 Mathematics Subject Classification. Primary 26A18; Secondary 54H20.

(C1984 American Mathematical Society $0002-9939 / 84 \$ 1.00+\$ .25$ per page 
$[0,1]$ which are in $C$. So when we write $x=b_{1} b_{2} b_{3} \cdots$, we always mean that $b_{i}=0$ or 2 for all $i \geqslant 1$, and $x$ is the real number whose (unique) ternary expansion is $b_{1} b_{2} b_{3} \cdots$. Let $A=a_{1} a_{2} a_{3} \cdots$. If the sequence $\left\langle a_{n}\right\rangle$ is periodic, i.e., $a_{i}=a_{m+i}$ for some $m \geqslant 1$ and all $i \geqslant 1$, then we write $A=\overline{a_{1} \cdots a_{m}}$. The notation $B=b_{1} b_{2} b_{3}$ $\cdots=c_{1} c_{2} \cdots c_{n} A$ will mean that $b_{i}=c_{i}$ for all $i=1,2, \ldots, n$, and $b_{n+k}=a_{k}$ for all $k \geqslant 1$. So $2 A$ is the real number whose ternary expansion is $2 a_{1} a_{2} a_{3} \cdots$. It does not mean 2 times $A$. For $A=a_{1} a_{2} a_{3} \cdots$, we let $A^{\prime}=a_{1}^{\prime} a_{2}^{\prime} a_{3}^{\prime} \cdots$ denote the real number whose ternary expansion is $\left(2-a_{1}\right)\left(2-a_{2}\right)\left(2-a_{3}\right) \cdots$. That is, $a_{i}^{\prime}=2-$ $a_{i}$ for all $i \geqslant 1$. With the above notation, we have $f(2 A)=A$ and $f(0 A)=A^{\prime}$.

Now we can state the following theorem. The proof is easy and omitted.

THEOREM. Let $A=a_{1} a_{2} a_{3} \cdots$. Then the following hold.

(a) $A$ is periodic with period $n$ (need not be minimal) if and only if $A=\overline{a_{1} \cdots a_{n}}$ with $a_{n}=2$, or $A=\overline{a_{1} \cdots a_{n} a_{1}^{\prime} \cdots a_{n}^{\prime}}$ with $a_{n}=0$.

(b) $A$ is eventually periodic with period $n$ (need not be minimal) if and only if $A=a_{1} \cdots a_{m} \overline{b_{1} \cdots b_{n}}$ or $A=a_{1} \cdots a_{m} \overline{b_{1}^{\prime} \cdots b_{n}^{\prime} b_{1} \cdots b_{n}}$ for some integer $m \geqslant 1$ and $b_{i}=0$ or 2 for all $i=1,2, \ldots, n$, with $b_{n}=a_{m}$.

(c) $A$ is asymptotically periodic with period $n$ (need not be minimal) if and only if $A=a_{1} \cdots a_{m n} \overline{b_{1} \cdots b_{n}}$ or $A=a_{1} \cdots a_{m n} \overline{b_{1}^{\prime} \cdots b_{n}^{\prime} b_{1} \cdots b_{n}}$ for some integer $m \geqslant$ 1 and $b_{i}=0$ or 2 for all $i=1,2, \ldots, n$, with $b_{n}=a_{m n}$.

(d) $A$ is recurrent if and only if, for every (large) integer $n>1$, there exists an integer $m(n)>1$ such that at least one of the following holds.

(1) If $a_{m(n)}=2$, then $a_{m(n)+i}=a_{i}$ for all $i=1,2, \ldots, n$.

(2) If $a_{m(n)}=0$, then $a_{m(n)+i}=a_{i}^{\prime}$ for all $i=1,2, \ldots, n$.

(e) $\bar{P}=\bar{R}=\Omega=C$, where $P, R, \Omega$ and $C$ are defined as before.

\section{REFERENCES}

1. W. A. Coppel, Maps on the interval, IMA Preprint Series, No. 26, University of Minnesota, 1983.

2. B.-S. Du, Are chaotic functions really chaotic, Bull. Austral. Math. Soc. 28 (1983), 53-66.

3. F. J. Fuglister, A note on chaos, J. Combin. Theory Ser. A 26 (1979), 186-188.

4. P. E. Kloeden, Chaotic difference equations are dense, Bull. Austral. Math. Soc. 15 (1976), 371-379.

5. T.-Y. Li and J. A. Yorke, Period three implies chaos, Amer. Math. Monthly 82 (1975), 985-992.

School of Mathematics, University of Minnesota, Minneapolis, Minnesota 55455

Current address: Institute of Mathematics, Academia Sinica, Nankang, Taipei, Taiwan 115, Republic of China 